\title{
LITERATUR
}

\section{Theorien unter der Lupe: \\ Der Forschungsstand zur europäischen Integration und den Internationalen Beziehungen}

\author{
Mareike Kleine*
}

Vor mehr als dreißig Jahren verglich der amerikanische Politikwissenschaftler Donald J. Puchala die Integrationsforschung mit dem Versuch, einen Elefanten durch Blinde beschreiben zu lassen. Da ein jeder einen anderen Part des Tieres untersucht, vermittelt letztlich auch jeder ein anderes Bild des Gegenstandes. ${ }^{1}$ Um ein Phänomen in allen Facetten $\mathrm{zu}$ verstehen, ist eine Perspektivenvielfalt daher notwendig und wünschenswert. Gerät ein solcher Pluralismus allerdings zum Selbstzweck, endet dies in einem babylonischen Stimmenwirrwarr, durch das - um bei dem Bild zu bleiben - eine möglichst vollständige Beschreibung des Elefanten in weite Ferne rückt. Um einem solchen Zustand vorzubeugen und einen fruchtbaren Dialog der Blinden zu ermöglichen ist es notwendig, den Forschungsstand und neuere Theorieentwicklungen regelmäßig zusammenzufassen und auf Gemeinsamkeiten und Unterschiede $\mathrm{zu}$ verweisen. Eben dies ist der Zweck der hier zur Rezension vorliegenden Sammelbände. Dabei fällt zunächst Folgendes auf: Erstens orientieren sich alle diese Bücher nicht mehr an gängigen Dichotomien wie beispielsweise Intergouvernementalismus versus Neofunktionalismus. Vielmehr wird jeweils von einem breiten Theorieverständnis ausgegangen, wel-
Siegfried Schieder und Manuela Spindler (Hrsg.): Theorien der Internationalen Beziehungen, Leske und Budrich: Stuttgart 2003, ISBN 38252-2315-9; 544 Seiten, 22,90€.

Gunther Hellmann, Klaus Dieter Wolf und Michael Zürn (Hrsg.): Die neuen Internationalen Beziehungen. Forschungsstand und Perspektiven in Deutschland. Nomos Verlagsgesellschaft: Baden-Baden 2003, ISBN 3-8329-0320-8; 614 Seiten, $29 €$.

Antje Wiener und Thomas Diez (Hrsg.): European Integration Theory, Oxford University Press: Oxford 2003, ISBN 0-19-925248-3; 306 Seiten, $18,99 €$.

Hans-Jürgen Bieling und Marika Lerch (Hrsg.): Theorien der europäischen Integration, Verlag für Sozialwissenschaften: Wiesbaden 2005, ISBN 3-8252-2554-2; 472 Seiten, 29,90 €.

ches sowohl für kausale als auch konstitutive Erklärungen offen ist und damit eine Vielzahl unterschiedlicher Fragestellungen zulässt. ${ }^{2}$ Dementsprechend erfolgt - zweitens - die Einteilung der Ansätze auch nicht ideengeschichtlich, sondern anhand epistemologischer und ontologischer Aspekte. So werden

* Mareike Kleine, Dipl. Pol., Wissenschaftliche Mitarbeiterin, Arbeitsstelle für Transatlantische Außen- und Sicherheitspolitik, Otto Suhr Institut für Politikwissenschaft, Freie Universität Berlin.

1 Donald J. Puchala: Of Blind Men, Elephants and International Integration, in: Journal of Common Market Studies, 3/1972, S. 267-284.

2 Daher werden im Folgenden die Begriffe Theorie und Ansatz synonym verwendet. Zu kausalen versus konstitutiven Erklärungen siehe Martin Hollis/Steve Smith: Explaining and Understanding International Relations, Oxford 1990 sowie John Gerard Ruggie: What Makes the World Hang Together? Neo-utilitarism and the Social Constructivist Challenge, in: International Organization, 4/1998, S. 855-885. 
zwar auf der einen Seite wesentliche Aspekte der Theorieentwicklung vernachlässigt. ${ }^{3}$ Auf der anderen Seite ermöglicht dies aber auch den Dialog zwischen Ansätzen der Internationalen Beziehungen (IB) und der vergleichenden Politikwissenschaft, der doch gerade im Bereich der europäischen Integration so sinnvoll und notwendig ist. So werden eben keine künstlichen Gräben mehr geschaffen, sondern auf Gemeinsamkeiten und feine Unterschiede hingewiesen. Die Notwendigkeit solcher Brückenschläge wird - drittens - von allen Herausgebern betont. Gerade in den deutschen IB, so Michael Zürn, sei dies inzwischen der Grundtenor, so dass man gar von einer „Venezianisierung" der Politikwissenschaft sprechen könne.

Während der Fokus der ersten beiden zu besprechenden Bücher auf dem Forschungsstand der IB liegt, stellen die beiden anderen Bände explizite Aufarbeitungen von Theorien der europäischen Integration dar. Im Folgenden sollen jeweils die Anlage der Bände diskutiert sowie beispielhaft einzelne Beiträge vorgestellt werden, welche zu einem tiefer gehenden Verständnis des Phänomens der europäischen Integration beitragen können.

\section{Perspektiven der Internationalen Beziehun- gen}

Weiterentwicklungen der IB-Theorien sind meist nach einiger Verzögerung auch in den Bereich der europäischen Integration übergeschwappt, so dass viele der Integrationstheorien überhaupt erst vor dem Hintergrund der in den IB herrschenden Debatten und Ausdifferenzierungen $\mathrm{zu}$ verstehen sind. Vor dem Hintergrund einer kaum noch zu überschauenden IB-Theorienlandschaft und dem Mangel insbesondere deutschsprachiger Einführungswerke sehen Siegfried Schieder und Manuela Spindler den unbedingten Bedarf eines Handbuchs mit Lehrbuchcharakter. Nach einer guten Darstellung des Zwecks und der Unterscheidungsmerkmale von Theorien wird dann leider etwas plötzlich und grob eine ,Vierteilung' der Theorienlandschaft in staatszentrierte Ansätze, gesellschaftszentrierte Ansätze, Ansätze aus der Internationalen Politischen Ökonomie, sowie Ansätze, welche das rationalistische Paradigma herausfordern, vorgenommen. Zwar hätten die Gründe für diese Einteilung durchaus genauer dargelegt werden können. Der Qualität des sonst empfehlenswerten Buches tut dies aber weiter keinen Abbruch, und so werden im Folgenden von einem jungen Autorenteam insgesamt 18 unterschiedliche Perspektiven auf fast durchgängig hohem Niveau vorgestellt. Dabei besitzen alle Kapitel die gleiche logische Struktur: Auf je 25-30 Seiten erfolgt zunächst eine Erläuterung der ideengeschichtlichen Wurzeln sowie die Darstellung des Ansatzes anhand der wesentlichen ReferenztheoretikerInnen. Nach der Auseinandersetzung mit interner und externer Kritik bildet schließlich ein didaktisch aufbereitetes Literaturverzeichnis den Abschluss. Im Zusammenhang mit einem Interesse an dem Phänomen der europäischen Integration ist unter anderem der Beitrag von Thomas Conzelmann zum Neofunktionalismus hervorzuheben, welcher eine ausgezeichnete Erörterung der Theorie von Ernst B. Haas sowie deren weiterer Umformulierungen darstellt. ${ }^{4}$ Auch Siegfried Schieders Darstellung des Neuen Liberalismus in den IB, wie er von einem positivistischen Wissenschaftsverständnis und in Abgrenzung zu früheren utopischen Formen von Andrew Moravcsik formuliert wurde, bietet eine sehr gute Grundlage für ein tiefer gehendes Verständnis des liberalen Intergouvernementalismus (LI). Darüber hinaus tragen auch die sehr verständlich geschriebenen Beiträge von Cornelia Ulbert zum Sozialkonstruktivismus sowie von Thomas Diez zu Postmodernen Ansätzen zu einer höheren Sensibilisierung von Debatten und Ausdifferenzierungen im Be-

3 Eine historische Darstellung der Integrationstheorieentwicklung bietet Ben Rosamond: Theories of European Integration, Houndmills 2000.

4 Siehe hierzu auch das kürzlich erschienene special issue des Journal of European Public Policy 2/2005. 
reich der europäischen Integration bei. Alles in allem ist es den Herausgebern gelungen, ein sehr gutes deutschsprachiges Handbuch mit Lehrbuchcharakter auf den Markt zu bringen, welches ohne Einschränkung zur Anschaffung empfohlen werden kann.

Vor dem Hintergrund zunehmender theoretischer und thematischer Ausdifferenzierung der „,neuen Internationalen Beziehungen“ fragen Gunther Hellmann, Klaus Dieter Wolf und Michael Zürn nach dem Forschungsstand und den Perspektiven der IB in Deutschland. Der aus einem Call for Papers hervorgegangene Sammelband soll einer systematischen Selbstreflexion der konzeptionellen und theoretischen Innovationen der letzten 15 Jahre dienen. In seiner Anlage gleicht er einem Handbuch, da zunächst im ersten Teil erkenntnistheoretische und konzeptionelle Grundlagen, im zweiten Teil klassische Themengebiete, und abschließend im dritten Teil neue Forschungsperspektiven diskutiert werden. Am Ende eines jeden Kapitels resümieren die Autoren jeweils mehr oder weniger erfolgreich die Bedeutung deutschsprachiger Beiträge in der internationalen Forschungslandschaft. Selbstbewusst, aber durchaus begründet, betonen die Herausgeber, dass sich die deutschen IB in den letzten 15 Jahren erheblich professionalisiert sowie sich ihr Stellenwert in Deutschland und weltweit verbessert habe. So scheinen allerdings auch andere nationale Forschungstraditionen irrelevant $\mathrm{zu}$ werden, und das Buch gleicht in seiner Anlage eher einem Vergleich von in Deutschland ansässiger Forschung mit den IB in den Vereinigten Staaten. Vor diesem Hintergrund stellt sich dann auch die Frage, ob dieser Vergleichsmaßstab nicht letztlich einen bias mit sich bringt, und der distinkte Charakter deutscher IB-Forschung dementsprechend überzogen dargestellt ist. Letztlich zählt aber, dass die einzelnen Beiträge von durchgängig hohem Niveau sind und eine gute Orientierung in dem Kanon deutscher IB-Forschung bieten. Dabei sind nicht alle Kapitel von unbedingter Relevanz für an dem Phänomen der europäischen Integration Interessierte. Auch sind die
Artikel nicht immer unmittelbar eingängig geschrieben, und so eignet sich das gesamte Buch eher für Fortgeschrittene und speziell an den IB interessierte Leser. Doch die Beiträge zur Metatheorie, aber vor allem die Darstellung des Regierens jenseits der Staatlichkeit durch Markus Jachtenfuchs geben einen instruktiven Einblick in Debatten, welche auch im Bereich der europäischen Integration Einzug gehalten haben.

\section{Das Mosaik der europäischen Integration}

Theorien der europäischen Integration sind systematische Reflektionen über den Prozess intensivierter politischer Kooperation, über die Entwicklung politischer Institutionen sowie über deren Ergebnisse. Von diesem weit angelegten Begriff ausgehend möchte der von Antje Wiener und Thomas Diez herausgegebene Band einen Überblick über eben jene Reflektionen geben. Dabei teilen sie die Beobachtung Puchalas, dass eine jede Theorie auf Grund unterschiedlicher ontologischer und epistemologischer Annahmen eine andere Perspektive auf das Phänomen der europäischen Integration bietet. Den Herausgebern zufolge bilden diese Perspektiven letztlich ein dauerhaft unvollständiges Mosaik, welches ein komplettes Bild der europäischen Integration aber zumindest erahnen lasse. Vorwiegend wurden die Referenztheoretiker selbst eingeladen, über ihren Beitrag zu diesem Mosaik zu reflektieren, und so versammelt das Buch eine Reihe namhafter Autoren wie, um nur einige zu nennen, Philippe Schmitter, Markus Jachtenfuchs und Beate Kohler-Koch. Nach einer Darstellung der ideengeschichtlichen Wurzeln soll der eigene Ansatz anhand eines typischen Falls und anhand eines Testfalls - der Erweiterung der Europäischen Union (EU) - erläutert werden. Die Erwartung der Herausgeber ist, dass sich auf Grund dieser unterschiedlichen Perspektiven auch die ausgewählten typischen Fälle sowie die an den Testfall angelegten Fragestellungen deutlich voneinander unterscheiden. 
In ihrem Einleitungskapitel identifizieren die Herausgeber zunächst drei Phasen der Entwicklung der Integrationstheorien: eine erklärende, eine analytische und eine konstruktive Phase. Die chronologisch sowie anhand der dominanten Fragestellung vorgenommene idealtypische Einteilung ist zwar in der Realität nicht immer durchzuhalten. Sie hilft aber doch, die einzelnen Steine des Theorienmosaiks zunächst grob voneinander abzugrenzen und den Band logisch zu strukturieren. Die erklärende Phase sei vorwiegend von Ansätzen aus dem Bereich der IB geprägt, welche den Prozess der Schaffung supranationaler Institutionen zu erklären versuchen. Erst in den 1980ern und mit der Einheitlichen Europäischen Akte rückten eben jene Institutionen selbst ins Blickfeld der Integrationsforschung. Der Fokus in dieser zweiten Phase liege immer stärker in der interdisziplinären Analyse des Regierens auf europäischer Ebene. In den 1990ern beginne schließlich die dritte, konstruktive Phase, in welcher sozialkonstruktivistische und kritische post-strukturalistische Ansätze immer stärker auch normative Fragen über die Konstruktion Europas aufwürfen.

Als Beitrag zur erklärenden Phase ist zunächst Frank Schimmelfennigs kritische Einführung in Andrew Moravcsiks LI hervorzuheben. Die Entscheidung zur Schaffung des Binnenmarktes wählt er zum einen als idealtypischen Fall aus, da hier alle Hintergrundvariablen für die Anwendung des LI gegeben seien. Zum anderen fragt er hinsichtlich des Testfalls nach den Gründen für die Entscheidung zur Erweiterung. Dabei gelingt es Schimmelfennig nicht nur, die Anwendung des LI zu demonstrieren, sondern auch auf Schwächen und Grenzen seiner Erklärungskraft hinzuweisen. Mark Pollacks Kapitel zu den „neuen Institutionalismen“, insbesondere zum rationalistischen und historischen Institutionalismus, stellt einen Beitrag zur analytischen Phase dar. ${ }^{5}$ Als die weltweit am dichtesten institutionalisierte internationale Organisation sei die Europäische Union nunmehr eine ausgezeichnete Projektionsfläche für Ansätze der vergleichenden Politikwissenschaft. Diese fragen vor allem nach (längerfristigen) Wirkungen dieser Institutionen auf Exekutive, Legislative und Judikative. Allerdings, bemerkt Pollack, seien die neuen Institutionalismen Theorien mittlerer Reichweite, welche Institutionen bereits voraussetzen, so dass die wesentlichen Gründe für Integration exogen bleiben müssten. Zudem basiere die rationalistische Variante auf äußerst strikten Annahmen und blende dadurch Aspekte aus, die vermutlich eher von soziologischen und sozialkonstruktivistischen Ansätzen erfasst werden könnten. Als Bestandteil der konstruktiven Phase habe der Sozialkonstruktivismus, so Thomas Risse in seinem Aufsatz, erst sehr spät einen spill-over aus den IB in den Bereich der europäischen Integration erfahren. Dennoch sei dieser Ansatz sehr schnell aus den metatheoretischen Wolken auf dem Boden der Empirie gelandet. Insbesondere drei wesentliche Aspekte trage der Sozialkonstruktivismus zu Fragen der europäischen Integration bei: Erstens lasse der Ansatz ein tiefer gehendes Verständnis der Europäisierung zu. Zweitens verweise er auf die konstitutiven Effekte europäischer Normen und Regeln auf Identitäten und Interessen der Akteure. Und drittens erlaube ein Fokus auf kommunikative Praktiken das Verständnis für die diskursive Konstruktion Europas und der Union.

Trotz der originellen Anlage des Buches und weiterer interessanter, doch hier nicht speziell hervorgehobener Beiträge, muss allerdings konstatiert werden, dass dem Band eine gewisse Kohärenz fehlt. Zum einen scheinen die Herausgeber große Mühe gehabt zu haben, manchem Referenztheoretiker beziehungsweise mancher Referenztheoretikerin das zu Grunde liegende Konzept nahe zu bringen. So

5 Siehe hierzu auch in längerer Form Mark A. Pollack: Theorizing The European Union: International Organization, Domestic Polity, or Experiment in New Governance?, in: Annual Review of Political Science 8/2005, S. 357-398. 
werden vereinzelt Gegenmodelle zum Theorienmosaik entworfen oder der Testfall Erweiterung schlichtweg ignoriert. Zum anderen kommt bei einer solchen Anlage natürlich auch die kritische Reflexion des eigenen Ansatzes deutlich zu kurz. So schwankt die Qualität der einzelnen Beiträge erheblich, und die im Schlusskapitel vorgenommene Gesamtschau, welche nach Ansicht der Herausgeber das Bild des Theorienmosaiks verdeutlichen sollen, wirkt eher künstlich. Als Lehr- und Handbuch ist der Sammelband daher nur bedingt zu empfehlen.

\section{Integrationstheorien für die Hosentasche}

Ganz anders das von Hans-Jürgen Bieling und Marika Lerch herausgegebene UTB-Taschenbuch: Hier sind die jungen Autoren alle , auf Linie gebracht“ und die Qualität der Artikel ist fast durchgängig hoch. Die Gliederung des Bandes orientiert sich allerdings etwas weniger an sich wandelnden Fragestellungen denn an historisch bedingten Theorieentwicklungen. Die Hauptblöcke bilden hier zunächst die klassischen Ansätze (wie Föderalismus und Neofunktionalismus), daraufhin Modifikationen (wie beispielsweise vom Intergouvernementalismus zum LI) sowie neue Perspektiven (wie das Konzept der Europäisierung), und letztlich neuere konstruktivistische, feministische und interdisziplinäre Impulse. Ähnlich dem von Schieder und Spindler herausgegebenen Band werden 15 Ansätze auf je circa 25 Seiten präsentiert, die alle der gleichen logischen Struktur folgen: Nach der Darstellung der ideengeschichtlichen Wurzeln werden anhand der ReferenztheoretikerInnen zunächst ontologische, epistemologische und normative Aspekte eines jeden Ansatzes erläutert. Der Diskussion interner Differenzierungen und Weiterentwicklungen folgt ebenfalls ein didaktisch aufbereiteter Literaturapparat.

In seiner Einführung in den klassischen Ansatz des Intergouvernementalismus Stanley Hoffmanns kann Hans-Jürgen Bieling mit gängigen Verkürzungen und Fehlinterpretati- onen aufräumen. Zwar teile der Intergouvernementalismus die realistische Prämisse der analytischen Priorität nationalstaatlicher Interessen. Jedoch würden bei Hoffmann viel stärker auch die historischen Kontextbedingungen, die Führungskraft nationaler Politiker sowie die Kontingenz von Entscheidungen betont. Zumal dieser selbst eine mechanische Kausalität für die Analyse der Weltpolitik ablehne, handele es sich beim Intergouvernementalismus viel eher um ein heuristisches Analyseraster zum besseren Verständnis konkreter empirischer Phänomene. Wolfgang Wagner stellt in seinem Beitrag einen Ansatz vor, welcher trotz seiner regelmäßigen Anwendung in Kompendien zu Integrationstheorien bisher selten eine eigenständige Erwähnung gefunden hat. Der aus der vergleichenden Politikwissenschaft stammende und hauptsächlich von Fritz Scharpf und Renate Mayntz entwickelte akteurszentrierte Institutionalismus versucht anhand spieltheoretischer Modelle komplexe Akteurskonstellationen darzustellen und dabei der Zweckrationalität von Akteuren ebensoviel Bedeutung zuzumessen wie den sie umgebenden Institutionen. Dies erlaubt auf der einen Seite die Systematisierung von typischen Kooperationshindernissen und damit eine Einschätzung der Wahrscheinlichkeit ihrer Überwindung. Auf der anderen Seite konnte auf Grund empirischer Beobachtungen dementsprechend überraschender Steuerungsfähigkeit dieser Ansatz wesentlich weiterentwickelt und mit anderen Perspektiven verbunden werden. Gerade für Politikwissenschaftler sehr aufschlussreich ist auch der im dritten Hauptblock vorgestellte Beitrag Ulrich Halterns zu Integration durch Recht (Integration Through Law - ITL). Er stellt die Forschungsarbeiten Joseph Weilers vor, welchem die Sensibilisierung der Politikwissenschaft für die Rolle des Rechts im europäischen Integrationsprozess zu verdanken ist. ITL werfe Licht auf zunächst unbeachtete, da formal-juristische Aspekte, die sich für die Entwicklung des inzwischen weltweit einzigartigen gemeinschaftlichen Rechtsraumes als bedeutend erwiesen haben. Allerdings operiere dieser Ansatz mit einem äußerst blassen 
und untertheoretisierten Rechtsbegriff, der aktuelle Debatten über Legitimität und Identität der Europäischen Union kaum zu fassen vermag.

Anders als bei dem zuvor diskutierten Band wird bei diesem Buch der kritischen Reflexion deutlich mehr Raum gegeben. Zwar geht dies bei einzelnen Autoren teilweise etwas zu Lasten der eigentlichen Darstellung. Dennoch vermögen die Anlage dieses Buches und die durchgängig verständlich geschriebenen Artikel ein sehr viel deutlicheres Bild des Phänomens der europäischen Integration zu entwerfen, so dass sich dieser Sammelband sowohl für die Lehre als auch als Handbuch für den Eigenbedarf ausgezeichnet eignet.

\section{Resümee}

Über einen Mangel an Ansätzen zur Beschreibung des Phänomens der europäischen Integration und der internationalen Beziehungen kann also nicht geklagt werden. Die Zusammenfassung dieses Theorien- und Perspektivenpluralismus war die Absicht der hier vorgestellten Sammelbände. Ausgehend von jeweils guten Einführungskapiteln, in denen die Herausgeber die entsprechenden Begriffe definieren sowie die Auswahl der Ansätze begründen, bieten alle vier hier vorgestellten Sammelbände durchaus gute Orientierungen in der komplexen Forschungslandschaft und Theoriewelt.

Die drei anfangs dargelegten Beobachtungen eines zunehmend weit angelegten Theoriever- ständnisses, einer an metatheoretischen Kriterien vorgenommenen Unterscheidung der einzelnen Ansätze, sowie einer sich daraus ergebenen Möglichkeit und Tendenz zu Brückenschlägen zwischen handlungstheoretischen Paradigmen und politikwissenschaftlichen Teilgebieten, stimmen durchaus optimistisch, spiegeln sie doch die realweltlichen Entwicklungen der letzten Jahre wieder. Vor dem Hintergrund einer immer schwieriger werdenden Trennung von Innen und $\mathrm{Au}-$ ßen erscheint eben auch eine Isolierung von vergleichender Politikwissenschaft und IB problematisch. Die diskutierten Bände lassen vermuten, dass sich die theoriegeleitete Forschung diesen Herausforderungen stellt und nicht ihre Bodenhaftung verlieren wird. Bei allen Brückenschlägen muss allerdings auch vor zwei Dingen gewarnt werden. Zum einen fällt auf, dass methodische Gesichtspunkte in allen diskutierten Bänden kaum Erwähnung finden. So bleibt zu befürchten, dass die zunehmende Auffächerung theoretischer Perspektiven mit einem methodischen anything goes einhergeht und die Beschreibungen der Blinden letztlich nichts zum Verständnis des Elefanten beitragen. Zum anderen bemängeln Klaus Dieter Wolf und Gunther Hellmann zu Recht die auffällige Geschichtsvergessenheit der IB-Forschung. Beide Aspekte dürfen aber nicht aus dem Rahmen der Forschungslandschaft fallen. Gerade hier, und insbesondere auf dem deutschsprachigen Markt, besteht weiterhin großer Bedarf an guten Büchern. 